\title{
Effects of compound traditional Astragalus and Salvia Miltiorrhiza extract on acute and chronic hepatic injury
}

\author{
Xiaoxiang Zhang ${ }^{1}$, Yan Yang ${ }^{2, *}$, Xin $\mathrm{Liu}^{3}$, Chao $\mathrm{Wu}^{2}$, Minzhu Chen ${ }^{2}$ \\ ${ }^{1}$ Department of Pharmaceutical Engineering, Hefei University of Technology, Hefei, Anhui, 230009, China; ${ }^{2}$ Department of \\ Pharmacology and Institute of Natural Medicine, Anhui Medical University, Hefei, Anhui, 230032, China; ${ }^{3}$ Therapeutics Research \\ Centre, University of Queensland, Princess Alexandra Hospital, Brisbane, Queensland, Qld 4102, Australia
}

\begin{abstract}
Previous reports showed that Compound Astragalus and Salvia miltiorrhiza extract (CASE), which was mainly composed of astragalosides, astragalus polysaccharide and salvianolic acids, inhibited hepatic fibrosis by mediating transforming growth factor- $\beta$ (TGF- $\beta$ )/Smad signaling. Our aim was to examine the effects of CASE on D-galactosamine (D-GalN) treated liver injury in mice and carbon tetrachloride $\left(\mathrm{CCl}_{4}\right)$-induced liver fibrosis in rats. CASE was administered to mice with D-GalN-induced liver injury and to rats with $\mathrm{CCl}_{4}$-induced liver fibrosis, respectively. Liver injury was routinely evaluated by relative liver weight, serum levels of ALT, AST, hyaluronic acid (HA), hepatic malondialdehyde (MDA) content, superoxide dismutase (SOD) activity, hydroxyproline (HYP) and histopathologic changes. Treatment of mice with CASE $(60,120$, and $240 \mathrm{mg} / \mathrm{kg}$, ig) significantly lowered ALT, relative liver weight, and MDA levels when compared with D-GalN treated mice. CASE $(120,240 \mathrm{mg} / \mathrm{kg})$ significantly lowered ALT, AST, $\mathrm{HA}, \mathrm{HYP}$, and MDA levels against $\mathrm{CCl}_{4}$ treated rats. Decreased SOD level was reversed with CASE treatment. Upon histopathological examination, CASE treatment had significantly inhibitory effect on the progression of hepatic fibrosis in rats. These results indicate that CASE might be effective in treatment and prevention of acute and chronic hepatic injury due to its antioxidant activity.
\end{abstract}

Keywords compound Astragalus and Salvia miltiorrhiza extract, hepatic injury, antioxidant

\section{INTRODUCTION}

Astragalus membranaceus, which has the effectiveness of "invigorate vital energy" and "invigorating splenic yang", has for centuries been considered a kind of tonic herbs in Chinese medicine and it has been used for treatment liver diseases alone or in many mixed herbs (Kang et al., 2004; Roxas and Jurenka, 2007). Salvia miltiorrhiza, which has the effectiveness of "nourishing the blood", "promoting blood flow" and "regulating qi to dissipate blood stasis", has also been used as a traditional medicine in China for centuries and considered a potent herb in Chinese medicine to treat liver diseases and cardiovascular diseases (Hu et al., 2007; Lin et al., 2006; Liu et al., 2000). In traditional Oriental medicine, combined use of herbal medicines for the treatment of liver diseases was a common therapeutic strategy in an attempt to enhance their anti-fibrotic effects and to minimize its side effects and toxicity. The combined effects of multidrug are related to constituents, doses, and ratio in combination. In order to obtain a more effective remedy and based on both traditional references and the results of our previous work mentioned above, combination of Astragalus membranaceus Bunge (Leguminosae) and Salvia miltiorrhiza Bunge (Lamiaceae), was applied to the treatment of liver fibrosis. We previously have investigated the effects of these three kinds of extract constituents (astragalosides, astragalus polysaccharides and salvianolic acid) and their

\footnotetext{
*Correspondence: Yan Yang

E-mail: yangyan276866@ sohu.com 31,2013

doi: http://dx.doi.org/10.5667/tang.2013.0004

(C)2013 by Association of Humanitas Medicine

TANG / www.e-tang.org
}

Received February 5, 2013; Accepted May 20, 2013; Published May optimal combination on the proliferation and collagen synthetic activity of serum stimulated hepatic stellate cells (HSC) by ${ }^{3} \mathrm{H}-\mathrm{TdR}$ and ${ }^{3} \mathrm{H}$-Proline incorporation tests (data not shown). The uniform design method was taken as a quantitative tool to optimize constituents, dosages and ratio in combination, to determine the nature of interaction among the constituents. The ratio of 70:1:1.85 in weight of crude herbs was found to be the optimal combination of Compound Astragalus and Salvia Miltiorrhiza extract (CASE) which was composed of astragalosides, astragalus polysaccharide and salvianolic acids.

The best dosage of CASE to inhibit the proliferation (inhibition ratio $60.3 \%$ ) and collagen synthesis (inhibition ratio $97.5 \%$ ) was $120.0 \mathrm{mg} / \mathrm{L}$. These in vitro results suggested that CASE might outstrip unity constituent. Standardized extracts of the Chinese herb prescription composed of Astragalus membranaceus or Salvia miltiorrhiza may be expected to synergistically exert the anti-fibrotic effects based on findings from our previous reports. Our previous study showed that CASE exerted anti-fibrotic effect in chronically injured liver by inhibiting TGF- $\beta /$ Smad signaling transduction (Yang et al., 2008), and inhibit HepG2 cell invasion by modulating TGF- $\beta /$ Smad signaling (Liu et al., 2010). These studies have resulted in considerable interest in CASE as a therapeutic agent in chronic liver disease. Although astragalosides, astragalus polysaccharide and salvianolic acids were individually reported as exhibiting anti-fibrotic effect (Zhang et al., 2003; Wu et al., 2003; Lin et al., 2006), combined use of the three extracts, as CASE for the treatment of liver fibrosis in rats, has not been reported in detail so far. Therefore, we hypothesized that the three extracts in combination might exhibited synergistic effect on the liver fibrosis in rats. In this study, D-GalN-induced liver injury in mice and $\mathrm{CCl}_{4}$-induced liver fibrosis in rats was used 
to investigate the effects of CASE on chronic and acute liver injury.

\section{MATERIALS AND METHODS}

\section{Plant materials and preparation of CASE}

The herbs Astragalus membranaceus and Salvia miltiorrhiza were purchased from Bozhou Crude Drug Market, Anhui, China. The medicinal materials were authenticated by Professor Jian-Li Zhou, Department of Medicinal Plant, Anhui College of Traditional Chinese Medicine. Voucher specimens were deposited at the Herbarium of the Anhui College of Traditional Chinese Medicine. The processes of extracting and preparing the three CASE components (astragalosides, astragalus polysaccharide and salvianolic acids) from the two herbs were described previously (Yang et al., 2008).

For in vivo experiment, the three extracted powders were dissolved in $0.5 \%$ sodium carboxymethylcellulose (CMC-Na) with a standard ratio (70:1:1.85) in weight of crude herbs.

\section{Chemicals}

$\mathrm{CCl}_{4}$ were purchased from Beijing Chemical Factory (China). D-galactosamine (D-GalN) was purchased from Chongqing Medical University (China). The kits for analyzing alanine aminotransferase (ALT), aspartate aminotransferase (AST), malondialdehyde (MDA), superoxide dismutase (SOD), and hydroxyproline (HYP) were purchased from Nanjing Jiancheng Institute of Biotechnology (China). Hyaluronic acid (HA) radioimmunoassay kits were purchased from Shanghai Navy Medical Institute (China). All other chemicals were of analytical grade and purchased locally.

\section{Animals and treatments}

Sprague-Dawley rats $(140-170 \mathrm{~g})$ of both sexes and Kunming strain mice (18 - $22 \mathrm{~g}$ ) of both sexes (Anhui Medical University, China) were under conditions of controlled temperature (25 \pm $2{ }^{\circ} \mathrm{C}$ ) and illumination (12 h light/dark cycle), and provided with rodent chow and water ad libitum. The rats were maintained in these facilities for at least 1 week before experiment. All procedures involving the use of laboratory animals were in accordance with the guidelines for the humane treatment of animals set by the Association of Laboratory Animal Sciences and the Center for Laboratory Animal Sciences at Anhui Medical University.

\section{Animal model of D-GalN induced liver injury and CASE treatment}

The mice were randomly divided into five groups with 10 mice in each group. Animals in group 1 (normal control group) and group 2 (D-GalN control group) were treated with a single daily dose of $0.5 \%$ CMC-Na solution. Animals of groups 3 - 5 were treated with CASE $(60,120$ and $240 \mathrm{mg} / \mathrm{kg})$ by gavage. All the treatments continued for 7 days. On the seventh day, animals of groups 2 - 5 were given a single dose of D-GalN ( $800 \mathrm{mg} / \mathrm{kg}$ in saline) by intraperitoneal injection $2 \mathrm{~h}$ after administration of CASE or $0.5 \%$ CMC-Na solution, while group 1 animals received normal saline instead of D-GalN. After $24 \mathrm{~h}$, the mice were bled by removing eyeball and the blood was collected. The serum was separated from blood and was used for assay of ALT. Mice livers were immediately removed after blood collection, and weighed. A portion of the liver was then homogenised in physiological saline solution and the homogenate was used for assay of MDA.

\section{Animal model of liver fibrosis and CASE treatment}

Sprague-Dawley rats were randomly divided into five groups (normal control group, $\mathrm{CCl}_{4}$ model group, and three CASE treatment groups with doses at 60,120 and $240 \mathrm{mg} / \mathrm{kg}$ ) with eight rats in each group. Experimental hepatic fibrosis was produced using the method of Iredale et al. (1998), with some modifications. Rats were subcutaneously injected with $12.5 \%$ $(\mathrm{v} / \mathrm{v}) \mathrm{CCl}_{4}$ in peanut oil, twice a week for 8 weeks. The amount of $\mathrm{CCl}_{4}$ administered was $0.5 \mathrm{~mL} / \mathrm{kg}$. The normal control group received equal amounts of olive oil. Rats in treatment groups were administered CASE in $0.5 \%$ CMC-Na solution by gavage once a day for 8 weeks, while the rats in normal control group and model group were administered with the same volume of $0.5 \%$ CMC-Na solution only. At the end of 8 weeks, blood was withdrawn from the abdominal aorta under ether anesthesia. After euthanasia, liver was removed immediately, and weighed, and relative liver weight was calculated as liver mass divided by body mass. The blood samples were allowed to clot for $45 \mathrm{~min}$ at room temperature. Serum was separated by centrifugation at $600 \times \mathrm{g}$ for $15 \mathrm{~min}$ and analyzed for various biochemical parameters including ALT, AST, and HA. A portion of the liver was homogenized in physiological saline solution. Homogenates were centrifuged $\left(1000 \times \mathrm{g}, 10 \mathrm{~min}, 4^{\circ} \mathrm{C}\right)$ and the supernatant was used for the assays of MDA, SOD and HYP content in liver. The activities of AST and ALT, the contents of HA, HYP, the levels of MDA and SOD, were determined using analysis kits (Nanjing Jiancheng Institute of Biotechnology, China). Furthermore, a portion of the left lobe of the rat liver was fixed in $10 \%$ formalin for $24 \mathrm{~h}$ and embedded in paraffin. Tissue sections $(5 \mu \mathrm{m})$ were used for histopathological examination.

\section{Histopathological examination}

Sections $(5 \mu \mathrm{m})$ were stained with hematoxylin and eosin (HE) for histopathological examination. Masson stain was used to determine liver fibrosis. Histological grade of hepatic fibrosis was determined by a semi-quantitative method according to the following scoring system (Li et al., 2003): 0, normal (no visible fibrosis); $1+$, fibrosis present (collagen fiber present that extends from portal triad or central vein to peripheral region); 2 + , mild fibrosis (mild collagen fiber present with extension without compartment formation); $3+$, moderate fibrosis (moderate collagen fiber present with some pseudo lobe formation); and $4+$, severe fibrosis (severe collagen fiber present with thickening of the partial compartments and frequent pseudo lobe formation).

\section{Statistical analysis}

The data were expressed as mean \pm S.D. Statistical analysis was performed by one-way analysis of variance (ANOVA), followed by student's t-test. Ranked data were compared using Ridit procedure. $p<0.05$ was considered as statistically significant.

Table 1. Effect of CASE on serum ALT, relative liver weight and tissue MDA of mice with D-GalN induced liver injury (mean \pm SD, $n=10$ )

\begin{tabular}{|c|c|c|c|c|}
\hline Group & Dose (mg/kg) & $\operatorname{ALT}(\mathbf{U} / \mathbf{L})$ & MDA $(\mathrm{nmol} / \mathrm{g})$ & Relative liver weight (\%) \\
\hline Control & 0 & $41.8 \pm 10.4$ & $153.0 \pm 23.3$ & $4.36 \pm 0.52$ \\
\hline Model & 0 & $155.1 \pm 41.2^{\# \#}$ & $325.0 \pm 84.9^{\# \#}$ & $6.16 \pm 0.87^{\# \#}$ \\
\hline \multirow[t]{3}{*}{ CASE } & 60 & $112.3 \pm 38.0^{*}$ & $256.9 \pm 45.6^{*}$ & $5.34 \pm 0.97^{*}$ \\
\hline & 120 & $103.4 \pm 36.7^{* * *}$ & $226.2 \pm 22.6^{* *}$ & $5.05 \pm 0.73^{* *}$ \\
\hline & 240 & $103.0 \pm 35.4^{* *}$ & $208.5 \pm 31.1^{* *}$ & $5.09 \pm 0.53^{* *}$ \\
\hline
\end{tabular}


Table 2. Effect of CASE on relative liver weight of hepatic fibrosis rats induced by $\mathrm{CCl}_{4}($ mean $\pm \mathrm{SD}, \mathrm{n}=8)$

\begin{tabular}{ccc}
\hline Group & Doses $(\mathbf{m g} / \mathbf{k g})$ & Relative liver weight $(\%)$ \\
\hline Control & 0 & $2.12 \pm 0.48$ \\
Model & 0 & $3.79 \pm 0.67^{\# \#}$ \\
CASE & 60 & $3.24 \pm 0.58$ \\
& 120 & $3.79 \pm 0.59$ \\
& 240 & $2.61 \pm 0.67^{*}$ \\
\hline${ }^{*} p<0.01$, as compared with normal control group. ${ }^{*} p<0.05$, as
\end{tabular}
compared with model group.

\section{RESULTS}

Protective effect of CASE on D-GalN induced liver injury in mice

Mice administered with D-GalN showed elevated levels of serum ALT, relative liver weight and tissue MDA, as compared with normal control group (Table 1). Treatment with CASE (60, 120 and $240 \mathrm{mg} / \mathrm{kg}$, i.g) significantly attenuated these levels.

\section{Effects of CASE on relative liver weight of hepatic fibrosis} rats

Relative liver weight was significantly increased after subcutaneous injection of $\mathrm{CCl}_{4}$ for 8 weeks, as compared with control animals. Orally administration of CASE at $240 \mathrm{mg} / \mathrm{kg}$ significantly lowered the relative liver weight (Table 2).

\section{Serum markers of hepatic fibrosis}

$\mathrm{CCl}_{4}$ administration resulted in significant $(p<0.01)$ rise in the levels of ALT, AST and HA when compared with the control group. Oral administration of CASE $(120 \mathrm{mg} / \mathrm{kg})$ was seen to lower significantly the elevated levels of HA. Treatment with CASE $(240 \mathrm{mg} / \mathrm{kg})$ caused significant decrease in serum transaminase activities (Table 3 ).

\section{Effect of CASE on SOD activity and the contents of MDA} and HYP in liver homogenates

As shown in Table 4, SOD level decreased significantly after $\mathrm{CCl}_{4}$ treatment $(p<0.01)$ compared to normal rats, while MDA and HYP contents of the liver significantly increased. CASE treatment (120 and $240 \mathrm{mg} / \mathrm{kg}$ ) significantly attenuated increase in MDA and HYP contents, and elevated SOD level in liver homogenate when compared with $\mathrm{CCl}_{4}$ treated rats.

\section{Effect of CASE on hepatic histology}

$\mathrm{CCl}_{4}$ treatment induced severe liver fibrosis compared to control group as indicated by Masson stain. CASE treatment at the dose of 120 and $240 \mathrm{mg} / \mathrm{kg}$ markedly reduced the average severity of $\mathrm{CCl}_{4}$ induced liver fibrosis (Table 5). Representative photographs of the liver morphology are shown in Fig. 1. The hepatic lobular architecture was normal and no histological abnormalities were observed in liver from normal control rats (Fig. 1A). In contrast, liver tissue from $\mathrm{CCl}_{4}$ treated rats (no CASE treatment) showed more steatosis, obvious collagen deposition, cell necrosis and inflammatory infiltration compared to those in normal control rats (Fig. 1B). CASE treatment (120 and $240 \mathrm{mg} / \mathrm{kg}$ ) markedly alleviated the degree of liver fibrosis as indicated by less collagen deposition and lowered inflammation (Fig. 1C and D).

\section{DISCUSSION}

$\mathrm{CCl}_{4}$ is a xenobiotic used extensively to induce oxidative stress. Chronic $\mathrm{CCl}_{4}$ treatment is frequently used in rats as an experimental model of hepatic fibrosis (Shyu et al., 2008). Liver fibrosis induced by $\mathrm{CCl}_{4}$ is associated with the exacerbation of lipid peroxidation (LP) and the depletion of antioxidant status. LP produces hepatocellular damage and enhanced production of fibrotic tissue (Tsukamoto et al., 1990). As a result of LP, cell membrane integrity is compromised and cell plasma enzymes such as ALT, AST leak out. In the present study, $\mathrm{CCl}_{4}$-induced increased serum AST and ALT were significantly suppressed by CASE treatment. MDA is one of the main LP products and its level reflects the degree of LP injury in hepatocytes. Increased levels of LP products have been associated with a variety of diseases in both humans and animal models (Karadeniz et al., 2009). In this study, we found that CASE significantly reduced the increase of MDA level caused by $\mathrm{CCl}_{4}$-treatment, which indicated that anti-fibrotic effect of CASE not only was exerted by inhibiting TGF- $\beta$ signal transduction (Yang et al., 2008) but also may be related to its antioxidant and free radical scavenging ability.

$\mathrm{D}-\mathrm{GalN}$ is indirect hepatotoxic agent. Although its metabolic product is not free radical, it could induce $\mathrm{Ca}^{2+} / \mathrm{Mg}^{2+}$

Table 3. Effect of CASE on serum levels of ALT, AST and HA in hepatic fibrosis rats induced by $\mathrm{CCl}_{4}($ mean $\pm \mathrm{SD}, \mathrm{n}=8$ )

\begin{tabular}{ccccc}
\hline Group & Doses $(\mathbf{m g} / \mathbf{k g})$ & ALT $(\mathbf{U} / \mathbf{L})$ & AST $(\mathbf{U} / \mathbf{L})$ & HA $(\boldsymbol{\mu g} / \mathbf{L})$ \\
\hline Control & 0 & $48.6 \pm 8.1$ & $23.0 \pm 8.7$ & $308.3 \pm 103.5$ \\
Model & 0 & $153.4 \pm 22.4^{\# \#}$ & $38.4 \pm 9.3^{\# \#}$ & $907.5 \pm 470.5^{\# \#}$ \\
CASE & 60 & $171.8 \pm 27.0$ & $43.0 \pm 8.3$ & $556.7 \pm 388.0$ \\
& 120 & $90.5 \pm 38.9^{* * *}$ & $41.1 \pm 7.6$ & $296.1 \pm 173.8^{* * *}$ \\
${ }^{\# \# \#} p<0.01$, compared with normal control group. ${ }^{*} p<0.05,{ }^{* *} p<0.01$, compared with model group.
\end{tabular}

Table 4. Effect of CASE on SOD activity and the contents of MDA and HYP in liver homogenates of rats treated with $\mathrm{CCl}_{4}(\mathrm{mean} \pm \mathrm{SD}, \mathrm{n}=8)$

\begin{tabular}{ccccc}
\hline Group & Doses $(\mathbf{m g} / \mathbf{k g})$ & SOD(U/mg protein) & MDA(nmol/mg protein) & HYP( $(\boldsymbol{\mu g} / \mathbf{g} \mathbf{l i v e r})$ \\
\hline Control & 0 & $329.9 \pm 57.9$ & $4.15 \pm 2.0$ & $154.5 \pm 72.2$ \\
Model & 0 & $231.2 \pm 77.4^{\# \#}$ & $10.68 \pm 4.7^{\# \#}$ & $423.7 \pm 119.0^{\# \#}$ \\
CASE & 60 & $282.3 \pm 36.6$ & $7.95 \pm 3.2$ & $279.7 \pm 111.6^{*}$ \\
& 120 & $306.4 \pm 43.7^{*}$ & $5.79 \pm 1.7^{*}$ & $208.7 \pm 137.5^{* *}$ \\
& 240 & $305.1 \pm 58.7^{*}$ & $5.81 \pm 2.1^{*}$ & $310.2 \pm 82.1^{*}$ \\
\hline${ }^{\# \#} p<0.01$, compared with normal control group. ${ }^{*} p<0.05,{ }^{* *} p<0.01$, compared with model group.
\end{tabular}

Table 5. Effects of CASE on the pathologic grading of $\mathrm{CCl}_{4}$-induced liver fibrosis in rats

\begin{tabular}{|c|c|c|c|c|c|c|c|}
\hline \multirow{2}{*}{ Group } & \multirow{2}{*}{ Dose (mg/kg) } & \multicolumn{5}{|c|}{ Severity score of hepatic fibrosis } & \multirow{2}{*}{$p$-value } \\
\hline & & $\mathbf{0}$ & 1 & 2 & 3 & 4 & \\
\hline Control & 0 & 8 & 0 & 0 & 0 & 0 & - \\
\hline Model & 0 & 0 & 0 & 1 & 2 & 5 & $0.001^{\# \#}$ \\
\hline \multirow[t]{3}{*}{ CASE } & 60 & 0 & 0 & 3 & 3 & 2 & 0.144 \\
\hline & 120 & 0 & 1 & 5 & 1 & 1 & $0.017^{*}$ \\
\hline & 240 & 0 & 1 & 3 & 3 & 1 & $0.040^{*}$ \\
\hline
\end{tabular}

Results are eight fields of vision. ${ }^{\# \#} p<0.01$, compared with normal control group. ${ }^{*} p<0.05$, compared with model group. 


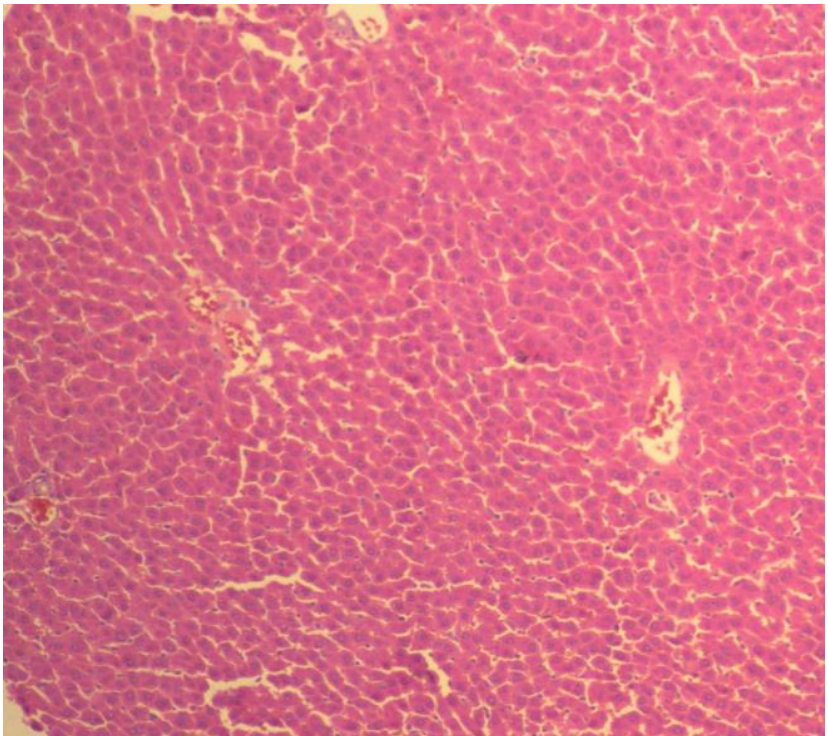

A

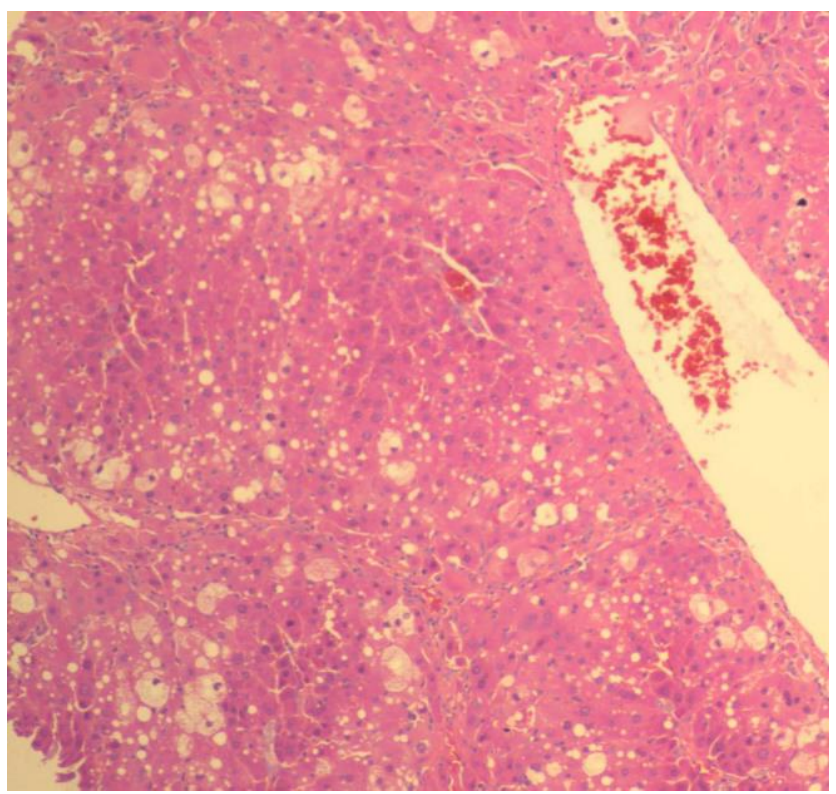

C

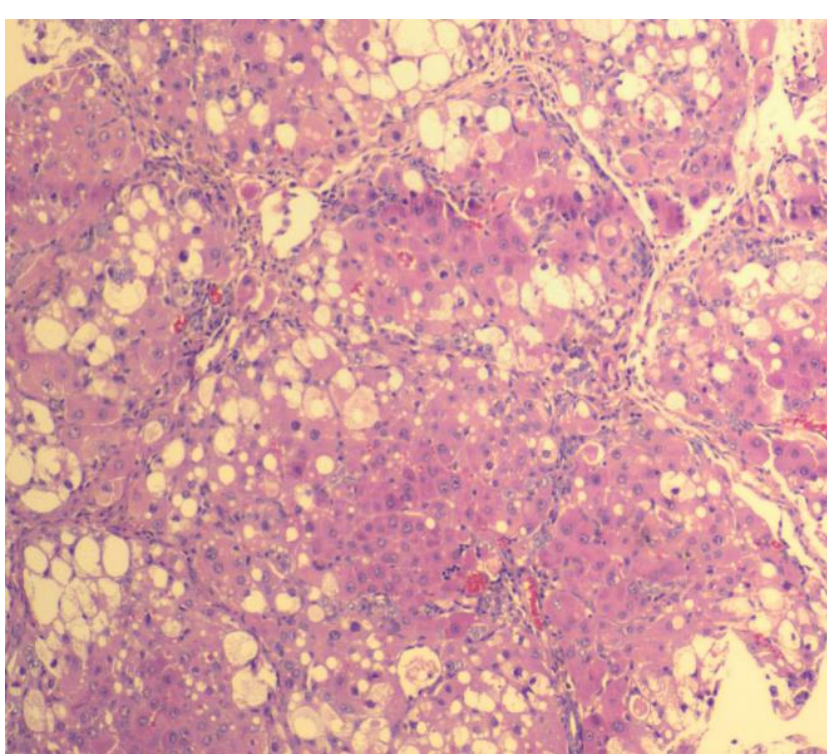

B

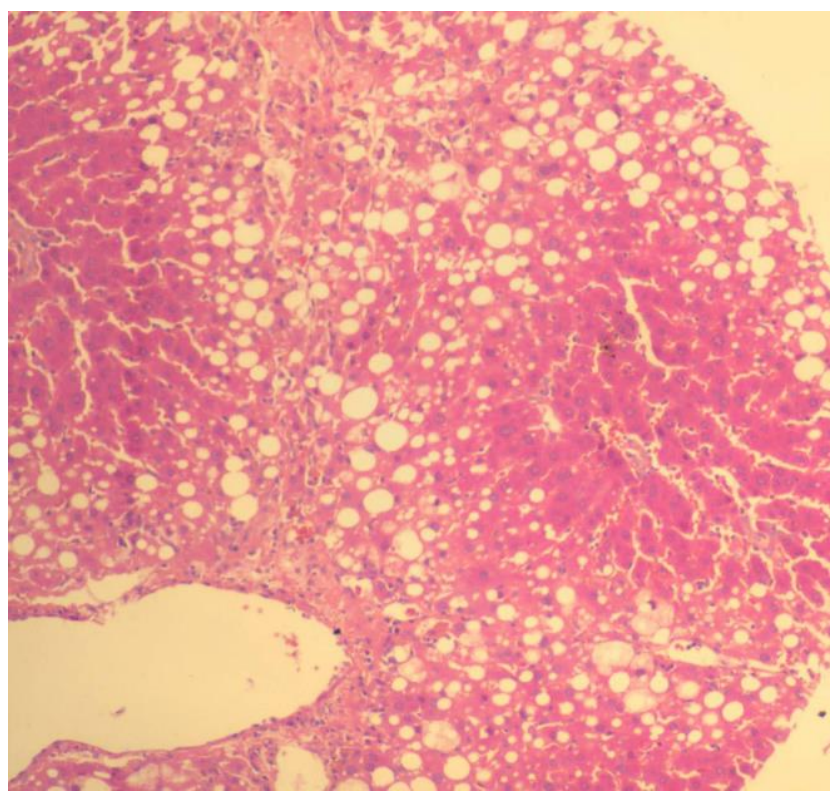

D

Fig. 1 Effect of CASE on the histological morphology of rats liver. (A) Normal control group; (B) Model group; (C or D) CCl 4 and CASE (120 or 240 $\mathrm{mg} / \mathrm{kg}$, ig.) treatment. HE stain. Magnitude $100 \times$

disproportion and conversion of xanthine dehydrogenase to xanthinoxidase, thus promote more oxygen free radicals to damage hepatocytes (Zhang et al., 1991). Anti-lipid peroxidation effect of CASE may contribute to its effect against D-GalN induced acute liver injury in mice.

In combined drug therapy, interactions among drugs in traditional medicine formulae are very complex. Two or more herbal medicines in combination are often more effective or powerful compared to the effect of single herb or its extract. Our previous results have shown that astragalus polysaccharides alone could weakly inhibit proliferation and collagen synthesis of HSC in vitro (Zhang et al., 2003). Astragalosides at the dose of $80 \mathrm{mg} / \mathrm{kg} \cdot \mathrm{d}(24 \mathrm{~g} / \mathrm{kg} \cdot \mathrm{d}$ as calculated by weight of Astragalus membranaceus) or Salvianolic acid $2.19 \mathrm{~g} / \mathrm{kg} \cdot \mathrm{d}$ in weight of Salvia miltiorrhiza alone could inhibit $\mathrm{CCl}_{4}$ induced liver fibrosis in rats (Huang et al., 2001). The best dosage of CASE found in this study for inhibition of $\mathrm{CCl}_{4}$ induced liver fibrosis in rats was $120 \mathrm{mg} /$ $\mathrm{kg} \cdot \mathrm{d}$ corresponding to $12.3 \mathrm{~g} / \mathrm{kg}$ in weight of Astragalus membranaceus and $0.32 \mathrm{~g} / \mathrm{kg}$ in weight of Salvia miltiorrhiza, respectively. It is obvious that CASE remarkably reduced the dosages of these two herbal medicines while showed similar therapeutic efficacy, which suggests that the combination of extracts is superior to the single component.

In our study, the CASE at the dose of $120 \mathrm{mg} / \mathrm{kg}$ showed stronger inhibitory effects on the contents of HYP and MDA in liver tissue and level of HA in serum than at the dose of 240 $\mathrm{mg} / \mathrm{kg}$. This finding was further confirmed by the histopathological examinations, namely, CASE treatment at the dose of $120 \mathrm{mg} / \mathrm{kg}$ was superior to CASE $(240 \mathrm{mg} / \mathrm{kg})$ in respect to CASE reducing the average severity of $\mathrm{CCl}_{4}$ induced liver fibrosis. The dosage of $240 \mathrm{mg} / \mathrm{kg}$ might increase hepatic metabolic load and thereby interfered its ameliorated action in liver lesion. The synergistic effects of two or more herbal medicines in combination on the liver fibrosis will be further studied.

An evidence of hepatic injury is leakage of cellular enzymes into the plasma. D-GalN at a dose of $800 \mathrm{mg} / \mathrm{kg}$ 
induced highly elevated levels of relative liver weight, serum ALT and tissue MDA in the untreated toxin control. When treated with CASE, a marked reduction of relative liver weight, ALT and MDA levels was observed. The likely hepatoprotective effect of CASE might be due to the stabilization of the liver cell membrane and the anti-lipid peroxidation.

In summary, in this study, the pharmacological effects of CASE were demonstrated in mice with D-GalN and in rats with $\mathrm{CCl}_{4}$-induced liver fibrosis, which indicates the possibility of using CASE for treatment and prevention of acute and chronic hepatic injury. In addition, mechanism of CASE effects was found to be associated with antioxidant and scavenging free radical ability.

\section{ACKNOWLEDGEMENTS}

We thank professor Qiang Wu, Anhui Medical University for his technical assistances in histological analysis.

This work was granted financial support from the National Natural Science Foundation of China (no.81073098) and the Scientific Research Foundation for Talent of Anhui Province (no.2008Z027).

\section{CONFLICT OF INTEREST}

All authors declare that there is no conflict of interest.

\section{REFERENCES}

Hu S, Chen S, Li X, Qin R, Mei Z. Antitumor effects of Chi-Shen extract from Salvia miltiorrhiza and Paeoniae radix on human hepatocellular carcinoma cells. Acta Pharmacol Sin. 2007;28:1215-1223.

Huang XZ, She SF, Wang GB. Experimental research of the various doses treatment groups of Salvia miltiorrhiza to prevent liver fibrosis induced with $\mathrm{CCl}_{4}$. Integr. Tradi and West Met J Hepatol. 2001;11:213-215.

Iredale JP, Benyon RC, Pickering J, McCullen M, Northrop M, Pawley S, Hovell C, Arthur MJ. Mechanisms of spontaneous resolution of rat liver fibrosis. Hepatic stelllate cell apoptosis and reduced hepatic expression of metalloproteinase inhibitors. J Clin Invest. 1998;102:538-549.

Kang H, Ahn KS, Cho C, Bae HS. 2004. Immunomodulatory effect of Astragali Radix extract on murine TH1/TH2 cell lineage development. Biol Pharm Bull. 2004;27:1946-1950.

Karadeniz A, Cemek M, Simsek N. The effects of Panax ginseng and Spirulina platensis on hepatotoxicity induced by cadmium in rats. Ecotoxicol Environ Saf. 2009;72:231-235.
Li C, Luo J, Li L, Cheng M, Huang N, Liu J, Waalkes MP. The collagenolytic effects of the traditional Chinese medicine preparation, Han- Dan-Gan-Le, contribute to reversal of chemical- induced liver fibrosis in rats. Life Sci. 2003;72:1563-1571.

Lin YL, Wu CH, Luo MH, Huang YJ, Wang CN, Shiao MS, Huand YT. In vitro protective effects of salvianolic acid B on primary hepatocytes and hepatic stellate cells. J Ethnopharmacol. 2006;105:215-222.

Liu J, Shen H, Ong CN. Salvia miltiorrhiza inhibits cell growth and induces apoptosis in human hepatoma HepG2 cells. Cancer Letters 2000;153:85-93

Liu X, Yang Y, Zhang X, Xu S, He S, Huang W, Roberts MS. Compound Astragalus and Salvia miltiorrhiza extract inhibits cell invasion by modulating transforming growth factor-b/Smad in HepG2 cell. J Gastroenterol Hepatol. 2010;25:420-426.

Roxas M, Jurenka J. Colds and influenza: a review of diagnosis and conventional, botanical, and nutritional considerations. Altern Med Rev. 2007;12:25-48.

Shyu MH, Kao TC, Yen GC. Hsian-tsao (Mesona procumbens Heml.) prevents against rat liver fibrosis induced by $\mathrm{CCl}_{4}$ via inhibition of hepatic stellate cells activation. Food Chem Toxicol. 2008;46:3707-3713.

Stachlewitz RF, Seabra V, Bradford B, Bradham CA, Rusyn I, Germolec D, Thurman RG. Glycine and uridine prevent D-galactosamine hepatotoxicity in the rat: role of Kupffer cells. Hepatology. 1999;29:737-745.

Tsukamoto H, Matsuoka M, French SW. Experimental models of hepatic fibrosis: a review. Semin Liver Dis. 1990;10:56-65.

Wu Q, Yang Y, Xue S, Zhang X, Zhou Y, Chen M. Effect of astragalosides on proliferation and collagen production of hepatic stellate cells in vitro. Chin Pharmacol Bull. 2003;19:892-895.

Yang Y, Yang S, Chen M, Zhang X, Zou Y, Zhang X. Compound Astragalus and Salvia miltiorrhiza Extract exerts anti-fibrosis by mediating TGF-beta/Smad signaling in myofibroblasts. J Ethnopharmacol. 2008;118:264-270.

Zhang XX, Yang Y, Chen MZ. Effect of total polysaccharide of Astragalus on proliferation and collagen production of HSC-T6 cells. Chin. J. Clin. Pharmacol. Ther (Chin). 2003;8:645-647. 